\title{
Effects of a prior short simulated training session on the subsequent occurrence of ventilatory thresholds
}

\author{
Gaël Guilhem ${ }^{a, b}$, Sylvain Dorela ${ }^{a}$ Francois Hug ${ }^{\text {a,b,* }}$ \\ a National Institute for Sports (INSEP), Paris, France \\ b University of Nantes, Nantes Atlantic Universities, Nantes, France
}

Received 12 July 2007; received in revised form 25 September 2007; accepted 7 November 2007

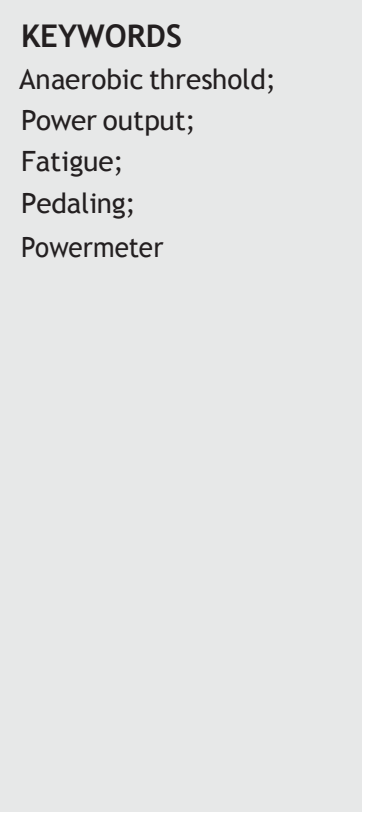

\begin{abstract}
Summary The concept of ventilatory thresholds (VTs) has been shown to be particularly useful to prescribe exercise intensities, yet, to date no study has examined the effects of previous submaximal exercise (i.e. a simulated training session) on the subsequent occurrence of VTs. We designed the present study to test the hypothesis that a previous short simulated training session induces an earlier VTs occurrence. Thirteen trained subjects perfomed two classical incremental tests on a cycle ergometer. The two tests were separated by a time period of $2-5$ days, and the second test was preceded by a 34-min simulated training session. The first and second VTs ( $\mathrm{VT}_{1}$ and $\mathrm{VT}_{2}$, respectively) were detected and expressed in their corresponding values of time, $\mathrm{V}_{2}$, and power output. The simulated training session did not modify $\mathrm{VT}_{1}$ occurrence. In contrast, $\mathrm{VT}_{2}$ was influenced by the simulated training session, showing a significant earlier occurence when $\mathrm{VT}_{2}$ was expressed in time $[-42(-72 ;-13) \mathrm{s}]$ and in power output $[-15(-25 ;-5) \mathrm{W}]$. Maximal power tolerated (MPT) was also significantly reduced by the previous exercise [ $-17(-27$; $-7) \mathrm{W}$. As a consequence, training intensities based on power output (associated with $\mathrm{VT}_{2}$ or in \%MPT) would be overestimated (i.e. higher metabolic state) after a short period of training session. Thus, doubt is cast on the pertinence of using the power output (or running speed by extension) for prescription of exercise during prolonged training sessions.

๑ 2007 Sports Medicine Australia. Published by Elsevier Ltd. All rights reserved.
\end{abstract}

\section{Introduction}

The concept of ventilatory thresholds (VTs) is closely linked in the literature to the concept of anaerobic threshold and respiratory compensation

\footnotetext{
* Corresponding author.

E-mail address: francois.hug@univ-nantes.fr (F. Hug).
}

point (respectively for the first and the second VTs). It has been shown to be particularly useful to assess the exercise tolerance of individuals with cardiorespiratory diseases. ${ }^{1}$ In the last few decades, this concept has engendered a great deal of interest from coaches, athletes and clinicians. In fact, numerous studies have shown that VTs could be considered a key indicator of aerobic 
aptitudes $^{2}$ and VTs can be used to measure the effects of endurance training. ${ }^{3}$ Moreover, VTs have been shown to be particularly useful to prescribe exercise intensities in order to optimize aerobic conditioning in pathological, ${ }^{4}$ sedentary ${ }^{5}$ or welltrained subjects. ${ }^{6}$ Indeed, various studies report that training at an intensity near the VTs induces a rightward shift of the lactate curve and a concomitant increase in VTs, either expressed in power output $(\mathrm{W})$ or oxygen uptake $\left(\mathrm{V}_{2}\right){ }^{3}$

The first and second VTs (i.e. $\mathrm{VT}_{1}$ and $\mathrm{VT}_{2}$, respectively) are classically determined during incremental exercise. Because several techniques based on visual inspection of respiratory gas exchange graphical plots have been described to measure VTs, their definitions are not always consistent in the literature. The most popular method to determine both $\mathrm{VT}_{1}$ and $\mathrm{VT}_{2}$ considers $\mathrm{VT}_{1}$ as the first point corresponding to an increase in the oxygen ventilatory equivalent $\left(\mathrm{EqO}_{2}\right)$; whereas a concomitant increase of $\mathrm{EqO}_{2}$ and the carbon dioxide ventilatory equivalent $\left(\mathrm{EqCO}_{2}\right)$ is considered evidence of $\mathrm{VT}_{2}$ occurrence. ${ }^{7}$ The continuous incremental exercise used for this VTs determination generally lasted for 8-12 min. ${ }^{8}$ However, depending on the type of training program employed for pathological subjects or the sport considered, training sessions can last more that $1 \mathrm{~h}$ (especially for endurance sports like road cycling or long/middle distance running). Thus, we wonder if the power output (or running speed) associated with VTs is altered as function of the exercise duration. In other words, is the exercise intensity prescribed to a subject using the conventional determination of VTs still valid after $1 \mathrm{~h}$ or more of training?

To the best of our knowledge, no study has examined the putative effects of a previous submaximal exercise (i.e. simulated training session) on the subsequent occurrence of VTs. Because numerous possible neural and humoral mechanisms may be postulated as generating VTs, ${ }^{9}$ it could be assumed that the accumulation of metabolites (e.g. potassium, lactate, ... .) occurring in muscles during a submaximal exercise could influence the subsequent occurrence of VTs.

The present study was designed to test the hypothesis that the power output associated to the ventilatory thresholds is reduced after a short simulated training session (i.e. $34 \mathrm{~min}$ ).

\section{Methods}

Thirteen healthy males volunteered to participate in this study [age: $26.2(23.5 ; 29.0)$ years, height: $179.0(176.1 ; 181.9) \mathrm{cm}$, body mass: 76.5 (71.9;
$80.9) \mathrm{kg}$, body mass index: $23.8(22.6 ; 25.1)]$ but were unaware of the nature of the experiment. Eight were ice hockey players and five were recreational sportsmen. All of them were instructed to refrain from intense physical activities during the 2 days prior to testing. They were informed of risk and discomfort associated with the experimental procedures before they gave their written consent to participate. The study was approved by the local ethics committee and was conducted in accordance with the Declaration of Helsinki.

Each subject performed the same incremental cycling test to exhaustion on an electrically braked cycle ergometer (Excalibur sport, Lode, The Netherlands), on two separate occasions. The two tests (i.e. control test and experimental test) were performed at the same period of the day and were separated by $2-5$ days. The experimental test was preceded by a 34-min of simulated cycling training session. A few days later, 6 out of the 13 subjects performed a third incremental cycling test identical to the control test (called retest), in order to assess the reproducibility of the VTs and maximal values. Because pedaling rate can influence the VTs occurrence the participants were asked, for all the incremental tests, to maintain the same pedaling rate $( \pm 2 \mathrm{rpm})$ freely chosen at the end of the warm-up period of the control test.

The incremental cycling test consisted of a 5-min warm-up period at $100 \mathrm{~W}$, after which the power output was increased by $20 \mathrm{~W} \mathrm{~min}^{-1}$ (Fig. 1A). This work rate increment was chosen to bring the subjects to the limit of their tolerance in about $10 \mathrm{~min}$, as recommended by Buchfuhrer et al. ${ }^{8}$ and in accordance with conventional protocols. ${ }^{10}$ Except pedaling rate, no other feedback $\left(\mathrm{V}_{2}\right.$, power output, time) was given to the participants. The progressive test was stopped when the pedaling rate could not be maintained.

After the control test ( $2-5$ days), the subjects performed the experimental test consisting of the same incremental cycling test preceded by a 34min simulated training session (Fig. 1B). They were asked to pedal at $100 \mathrm{~W}$ for 5 -min before performing a 3-min bout at the power output corresponding to $\mathrm{VT}_{1}$ (determined during the control test). After a 5-min active recovery period at $140 \mathrm{~W}$, they performed two bouts of 3-min (separated by 10-min at $140 \mathrm{~W}$ ) at the power output corresponding to $\mathrm{VT}_{2}$ (determined during the control test). Participants then observed a final 5-min recovery period at $100 \mathrm{~W}$ before starting the same incremental cycling test performed during the control test. Overall, the intensity distribution of the simulated training session (i.e. $73.6 \%$ below $\mathrm{VT}_{1}, 8.8 \%$ at $\mathrm{VT}_{1}$ and $17.6 \%$ at $\mathrm{VT}_{2}$ ) was very similar to the classical training dis- 

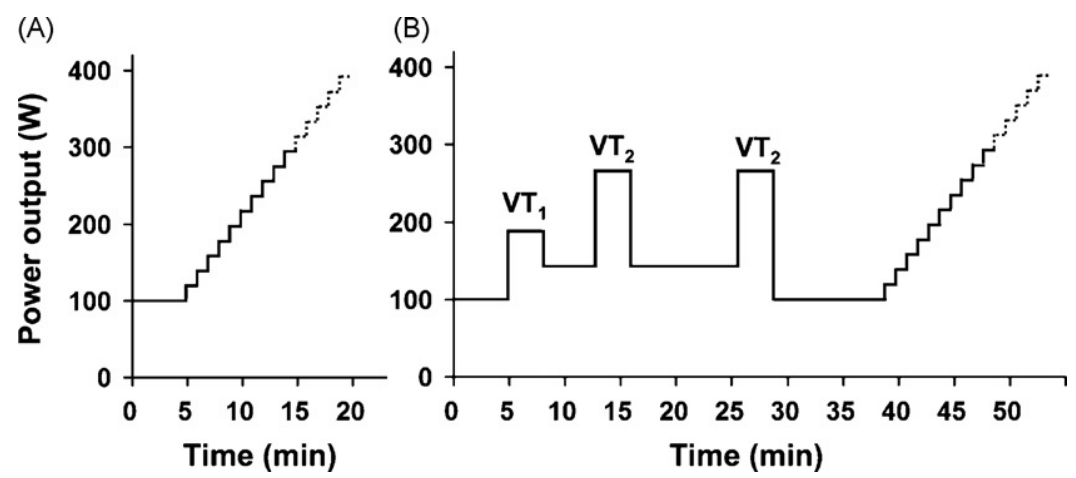

Figure 1 Schematic representation of the experimental design for the control test (A) and the experimental test (B). $\mathrm{VT}_{1}$, first ventilatory threshold; $\mathrm{VT}_{2}$, second ventilatory threshold.

tribution reported in a group of well-trained junior cross-country skiers ${ }^{11}$ or cyclists. ${ }^{12}$ In order to prevent dehydration during this protocol, participants were hydrated twice (at 21 and $34 \mathrm{~min}$ ) with $250 \mathrm{~mL}$ of a commercial solution (Powerade, United Sates).

A gas exchange analyzer $\left(\mathrm{K}_{4} \mathrm{~b}^{2}\right.$, Cosmed ${ }^{\circledR}$, Italy) measured breath-by-breath data of $\dot{\mathrm{V}}_{\mathrm{E}}, \dot{\mathrm{V}}_{2}, \mathrm{VCO}_{2}$, and calculated the ventilatory equivalents for $\mathrm{O}_{2}$ $\left(\mathrm{EqO}_{2}\right)$ and $\mathrm{CO}_{2}\left(\mathrm{EqCO}_{2}\right)$. Satisfactory test-retest reliability of this analyzer was previously demonstrated by various authors. ${ }^{13,14}$ All the respiratory and ventilatory values were averaged every $10 \mathrm{~s}$.

$\dot{\mathrm{V}} \mathrm{O}_{2}$ peak and $\dot{\mathrm{V}}_{\mathrm{Emax}}$ were defined as the average $\dot{\mathrm{V}} \mathrm{O}_{2}$ and $\dot{\mathrm{V}}_{\mathrm{E}}$ attained in the last $30 \mathrm{~s}$ of the tests. ${ }^{15}$ Maximal aerobic power (MAP) was defined as the lowest associated power that elicited the $\mathrm{V}_{2}$ peak. Maximal power tolerated (MPT) was defined as the power corresponding to the last entirely completed stage.

According to Reinhard et al. ${ }^{7} \mathrm{VT}_{1}$ corresponds to the time value at which $\mathrm{EqO}_{2}$ exhibited a systematic increase without a concomitant increase in $\mathrm{EqCO}_{2} . \mathrm{VT}_{2}$ was determined by using the criteria of an increase in both $\mathrm{EqO}_{2}$ and $\mathrm{EqCO}_{2}$. Two trained independent observers blindly detected $\mathrm{VT}_{1}$ and $\mathrm{VT}_{2}$ following the criteria previously described. The results obtained by the two observers were averaged and VTs were expressed in their correspondent values of time, \% of total test time, $\dot{\mathrm{V}}_{2}, \%$ of $\dot{\mathrm{VO}}_{2}$ peak, power output and \% of MPT.

All analyses were performed with Instat $^{\circledR}$ (GraphPad software, USA) and Medcalc ${ }^{\circledR}$ (Medcalc, Belgium) software for Windows. Data were first tested for normality using a Kolmogorov-Smirnov test. The results are expressed as mean and 95\% confidence intervals. Inter-observers reliability for VTs determination was assessed using the kappa coefficient. A paired-t-test was used to compare VTs and maximal values between control and experimental tests. Differences were considered significant when probability $(p)$ of a type I error was below $5 \%$. The intra-class correlation coefficient (ICC, ratio between intra-class variance and total variance) was calculated in order to test the reproducibility of VTs and maximal values between the control test and retest (for the 6 subjects tested). ICC represents a relative and dimensionless reliability variable. In addition to ICC, the standard error of measurement or typical error (S.E.M.) was determined to estimate an absolute measure of reliability.

\section{Results}

The 34-min simulated training session elicited a mean power output of $160(155 ; 164) \mathrm{W}$ and a mean oxygen uptake of $33.1(31.4 ; 34.7) \mathrm{mL} \mathrm{min}^{-1} \mathrm{~kg}^{-1}$. These data correspond to $45.9(44.6 ; 47.2) \%$ of MPT and $60.4(57.2 ; 63.6) \%$ of $\dot{\mathrm{V}}_{2}$ peak. Respiratory and ventilatory values before each incremental test were averaged during the last minute at $100 \mathrm{~W}$ (just before the beginning of the first workload increment): $\dot{\mathrm{V}}_{\mathrm{E}}, \dot{\mathrm{V}}_{2}$ and $\mathrm{EqCO}_{2}$ values were significantly higher for the experimental test condition (see supplemental file, Appendix A).

MAP, MPT, $\dot{\mathrm{V}}_{\text {Emax }}$ and total test time significantly decreased between control test and experimental test (Table 1). However, $\dot{\mathrm{V}}_{2}$ peak was unaffected by the previous simulated training session.

The figure attached to the electronic version of this article (Appendix A) depicts an individual example of VTs determination using the ventilatory equivalents method. The Kappa test revealed that the intra-observers reliability of the VTs determination was almost perfect $(K=0.91$ and 0.89 for $\mathrm{VT}_{1}$ and $\mathrm{VT}_{2}$, respectively). The simulated training session (i.e. previous submaximal exercise) did not modify $\mathrm{VT}_{1}$ occurrence when expressed in time, power output, $\dot{\mathrm{V}} \mathrm{O}_{2}$, \% of MPT, \% of $\dot{\mathrm{V}}_{2}$ peak, and 
Table $1 \quad \mathrm{VT}_{1}$ and $\mathrm{VT}_{2}$ and maximal values obtained during the control test and the experimental test

\begin{tabular}{|c|c|c|c|}
\hline & Control test $n=13$ & Experimental test $n=13$ & Mean difference \\
\hline \multicolumn{4}{|l|}{ First ventilatory threshold } \\
\hline$\dot{\mathrm{V}} \mathrm{O}_{2}\left(\mathrm{~mL} \mathrm{~min}^{-1} \mathrm{~kg}^{-1}\right)$ & $34.8(31.9 ; 37.7)$ & $36.1(33.3 ; 38.9)$ & $1.4(-1.8 ; 4.5)$ \\
\hline$\% \dot{\mathrm{VO}}_{2}$ peak & $62.8(60.3 ; 65.3)$ & $67.2(63.5 ; 70.8)$ & $4.3(-0.6 ; 9.3)$ \\
\hline Time (s) & $330(283 ; 378)$ & $308(267 ; 348)$ & $-23(-64 ; 19)$ \\
\hline$\%$ Total test time & $40.3(36.1 ; 44.5)$ & $40.4(36.0 ; 44.8)$ & $0.09(-5.60 ; 5.78)$ \\
\hline Power output (W) & $192(176 ; 208)$ & $183(169 ; 197)$ & $-9(-24 ; 5)$ \\
\hline$\%$ Maximal power tolerated & $55.9(52.8 ; 59.1)$ & $56.2(52.8 ; 59.6)$ & $0.3(-4.1 ; 4.6)$ \\
\hline \multicolumn{4}{|l|}{ Second ventilatory threshold } \\
\hline$\dot{\mathrm{v}} \mathrm{O}_{2}\left(\mathrm{~mL} \min ^{-1} \mathrm{~kg}^{-1}\right)$ & $45.7(41.7 ; 49.7)$ & $45.3(41.8 ; 48.7)$ & $-0.4(-3.7 ; 2.8)$ \\
\hline$\% \dot{\mathrm{VO}}_{2}$ peak & $82.4(79.7 ; 85.1)$ & $84.0(80.4 ; 87.7)$ & $1.7(-2.4 ; 5.8)$ \\
\hline Time (s) & $568(526 ; 610)$ & $525(476 ; 575)$ & $-42(-72 ;-13)^{*}$ \\
\hline$\%$ Total test time & $70.0(67.3 ; 72.7)$ & $69.1(64.8 ; 73.4)$ & $-0.9(-6.1 ; 4.3)$ \\
\hline Power output (W) & $272(257 ; 287)$ & $257(239 ; 274)$ & $-15(-25 ;-5)^{*}$ \\
\hline$\%$ Maximal power tolerated & $79.4(76.4 ; 82.5)$ & $78.9(75.1 ; 82.5)$ & $-0.6(-4.6 ; 3.4)$ \\
\hline \multicolumn{4}{|l|}{ Maximal values } \\
\hline$\dot{\mathrm{V}} \mathrm{O}_{2}$ peak $\left(\mathrm{mL} \mathrm{min}^{-1} \mathrm{~kg}^{-1}\right)$ & $55.4(51.3 ; 59.4)$ & $54.2(49.3 ; 59.0)$ & $-1.2(-4.5 ; 2.1)$ \\
\hline$\dot{\mathrm{V}}_{\mathrm{Emax}}\left(\mathrm{L} \min ^{-1}\right)$ & $171(157 ; 185)$ & $161(147 ; 175)$ & $-10(-21 ;-1)^{*}$ \\
\hline Total test time $(\mathrm{s})$ & $811(764 ; 859)$ & $761(705 ; 818)$ & $-50(-84 ;-16)^{*}$ \\
\hline Maximal power tolerated (W) & $343(326 ; 360)$ & $326(307 ; 345)$ & $-17(-27 ;-7)^{*}$ \\
\hline Maximal aerobic power (W) & $338(322 ; 354)$ & $325(305 ; 344)$ & $-14(-25 ;-2)^{*}$ \\
\hline
\end{tabular}

in \% of total test time $(p>0.05)$ (Fig. 2A; Table 1$)$. In contrast, $\mathrm{VT}_{2}$ was influenced by the simulated training session, showing a significant earlier occurrence during the experimental test when $\mathrm{VT}_{2}$ was expressed in time (corresponding to a decrease of $7,6 \%$ ) (Table 1) and power output (Fig. 2B). No change was found when $\mathrm{VT}_{2}$ was expressed in $\mathrm{VO}_{2}$, \% of MPT, \% of $\dot{\mathrm{V}}_{2}$ peak and in \% of total time (Table 1).

Appendix A (supplemental file) demonstrates good reproducibility between control test and retest ( $n=6$; ICC ranged from 0.79 to 1.00 ) for both VTs and maximal values.

\section{Discussion}

The present study reports for the first time that a short simulated training session alters the subsequent maximal performance and the occurrence of the second ventilatory threshold. Indeed, $\mathrm{VT}_{2}$ appeared at a significant lower output when the incremental exercise was preceded by the short simulated training session.

On a methodological point of view, numerous authors reported higher test-retest correlation scores using ventilatory equivalents to detect the VTs. ${ }^{3,7,10}$ Caiozzo et al. ${ }^{10}$ showed that the use of different ventilatory variables does not add significant improvement in term of validity in the estimation of $\mathrm{VTs}$ based on $\mathrm{EqO}_{2}$ and $\mathrm{EqCO}_{2}$. For this reason, VTs were only determined with the ventilatory equivalents method described by Reinhard et al. ${ }^{7}$ In this view, the high ICC and low S.E.M. values obtained between the control test and retest $(n=6$, see Appendix A) suggest good reproducibility for $\mathrm{VT}_{1}$ and $\mathrm{VT}_{2}$. Furthermore, the Kappa test reported an almost perfect intra-observers reliability suggesting a good precision of our VTs determination.

Data of the present study mainly shows the earlier occurrence of $\mathrm{VT}_{2}$ after a short simulated training session. To the best of our knowledge, no previous study has focused on the effect of a previous sub-maximal exercise on $\mathrm{VT}_{2}$ occurrence and only few studies are available concerning $\mathrm{VT}_{1}$. Black et al. ${ }^{16}$ reported that a previous exercise induces a later occurrence of $\mathrm{VT}_{1}$ (i.e. named aerobic threshold by the authors). It could be explained by the recent results reported by Raymer et al., ${ }^{17}$ showing a delayed onset of intracellular acidosis during incremental exercise when preceded by a bout of heavy-intensity exercise 6 min prior. However, the previous exercise performed in these studies (i.e. $10 \mathrm{~min}$ at $\mathrm{VT}_{1}$ and $6 \mathrm{~min}$ at $70 \%$ of peak power output for Black et al. ${ }^{16}$ and Raymer et al. ${ }^{17}$, respectively) result in a "warm-up" effect and not in a simulated training session. We would suggest that our 34-min session might have been too long to benefit from the positive effect described by these authors. Later, Marles et al. ${ }^{18}$ showed that intense previous 

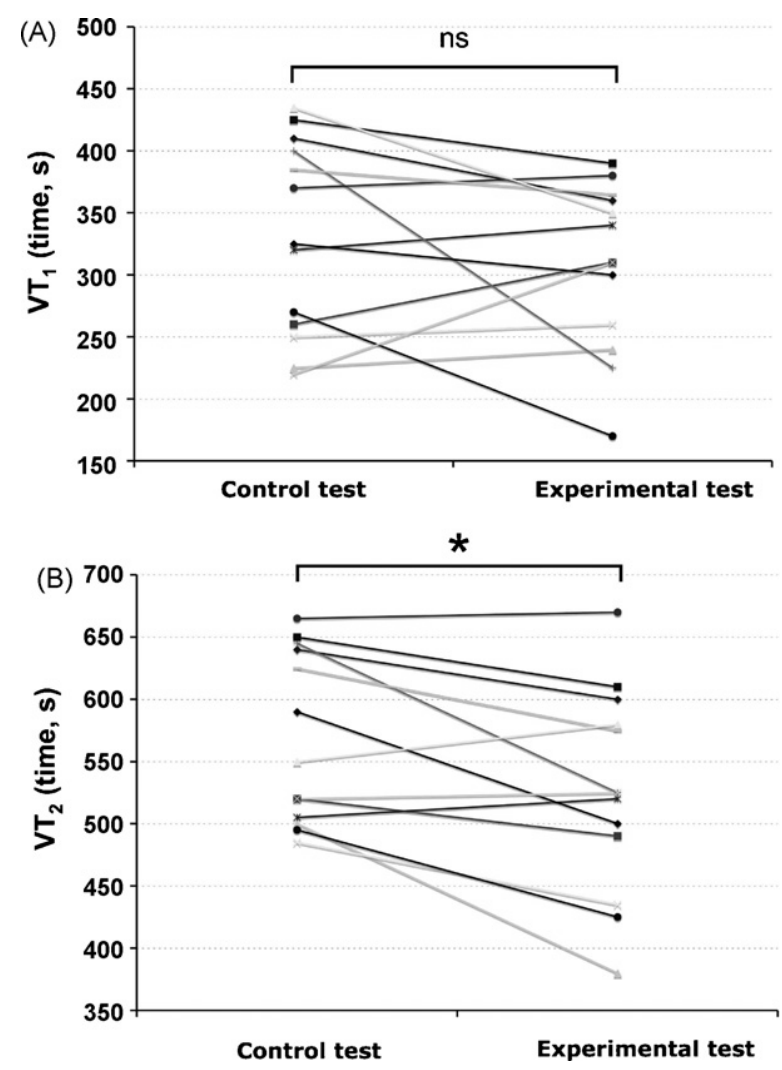

Figure 2 Time associated to the first (A) and second (B) ventilatory threshold is depicted for each participant for the control and the experimental test. Ns: no significant difference. ${ }^{*} p<0.05$.

exercise (i.e. $3 \mathrm{~min}$ at $35 \mathrm{~W}$ followed by $6 \mathrm{~min}$ at $90 \%$ of $\dot{\mathrm{VO}}_{2}$ peak and $6 \mathrm{~min}$ at $35 \mathrm{~W}$ ) did not affect $\mathrm{VT}_{1}$. Although it confirms our results (i.e. no effect of the simulated training session on $\mathrm{VT}_{1}$ ), the duration of exercise remains lower than that achieved in our study (15-min vs. 34-min).

Wasserman and Mcllroy ${ }^{1}$ first explicitly developed the concept that a critical threshold (i.e. called anaerobic threshold) exists at which there is a sudden increase in anaerobic metabolism leading to the beginning of lactate production and accumulation in the muscle. However, numerous recent studies demonstrated that anaerobic threshold does not correspond to the beginning of lactate production but is most likely a period of time during which lactate production begins to exceed the body's capacity to remove it (for review see Ref. 9). Hence, it seems more appropriate to replace the term "anaerobic threshold" by "ventilatory threshold". It was also experimentally shown that stimulation of chemoreceptors by lactic acid cannot be solely responsible for the VTs occurrence. ${ }^{19,20}$ Moreover, other studies suggested that direct stimulation of group IV muscle affer- ents by sufficient accumulation of metabolites has occurred in a fatigued muscle could play a key role in the hyperventilation ${ }^{21}$ and in the occurrence of VTs. ${ }^{20,22}$ Overall, it can be postulated that fatigue would occur during the short simulated training session and that the 10 -min of active recovery at $100 \mathrm{~W}$, between the end of the last bout at $\mathrm{VT}_{2}$ and the beginning of the workload increment, was not sufficient for a complete recovery. This would induce the earlier occurrence of $\mathrm{VT}_{2}$ and decrease the performance since our participants exhibited lower maximal values. According to Appendix A (supplemental file), it appears that baseline $\dot{\mathrm{VO}}_{2}$ (i.e. during the last minute at $100 \mathrm{~W}$ ) was higher on average by $160 \mathrm{~mL} / \mathrm{min}$ for the experimental test compared to the control test. It suggests a lower gross efficiency that would be caused by a greater accumulation of metabolites. The fact that $\mathrm{VT}_{1}$ was not significantly different from the control test $(192 \mathrm{~W}$ vs. $183 \mathrm{~W})$ could be an issue of statistical power (type II error) rather than physiological explanation or due to the greater variability of $\mathrm{VT}_{1}$ determination. Moreover, it should be kept in mind that the difference between control and experimental test found for $\mathrm{VT}_{2}$ is small (i.e. $15 \mathrm{~W}$ ) regarding the moderate accuracy of some powermeters. However, we can postulate that this difference would be higher after a longer training session.

Determination of the specific intensities associated with VTs is very useful in prescribing adequate loads for training programs. Several other studies have demonstrated the efficacy of exercise training at $\mathrm{VT}_{1}$ in the treatment of patients suffering from chronic heart failure ${ }^{23}$ or chronic obstructive pulmonary disease (COPD). ${ }^{24}$ Despite the fact that training at higher intensities has minimal benefit over the minimum intensity in sedentary and pathological subjects, it is crucial for conditioned subjects. Indeed, various studies have shown that further improvements in performance of trained subjects can only be achieved using high intensity interval training. ${ }^{25}$ Thus, numerous training protocols including exercises performed at a power output or speed corresponding to $\mathrm{VT}_{2}$ were developed and recommended. ${ }^{6,26}$ For instance, Dufour et al. ${ }^{26}$ reported a training session in highly trained male runners which consisted of a 10-min warmup period at $60 \% \mathrm{~V}_{2}$ peak, followed by two 20 -min periods at running speed corresponding to $\mathrm{VT}_{2}$, separated by 5 -min recovery at $60 \% \mathrm{VO}_{2}$ peak. Taking into consideration the results of our study, it can be reasonably hypothesized that the last bout at $\mathrm{VT}_{2}$ (after 35-min of exercise) was really performed at an higher intensity than $\mathrm{VT}_{2}$. Thus, we can postulate that an exercise prescribed in respect to power 
output associated with $\mathrm{VT}_{2}$ would be difficult to perform at the end of a training session. In support of this idea, Lepretre et al. ${ }^{27}$ recently showed that heavy exercise (i.e. intensity fixed between the lactate threshold and $\dot{\mathrm{VO}}_{2}$ peak) can be maintained two times longer under control of $\dot{\mathrm{V}}_{2}$ compared to power output.

On the other hand, as mentioned in the review of Laursen and Jenkins ${ }^{28}$ training intensity can also be based on a percentage of MPT, independently of VTs. Because the results clearly showed that MPT is reduced after a short simulated training session, it could also be assumed that such intensities are overestimated after a short period of the training session.

Lastly, various authors ${ }^{29}$ used VTs in order to study the distribution of training/competition intensities. For instance, based on power output or speed recordings, they distinguished time spent at (1) low intensity $<V^{2} T_{1}$, (2) moderate intensity between $\mathrm{VT}_{1}$ and $\mathrm{VT}_{2}$ and (3) high intensity $>\mathrm{VT}_{2}$. In some cases, the training/competition duration can exceed $6 \mathrm{~h}$ (i.e. some stages of the Tour de France). Because we showed that exercise intensity associated with $\mathrm{VT}_{2}$ decreases after a short training session, we could assume that this specific intensity also decreases during these training/competition periods. It could therefore induce some alterations in the distribution of the total time spent in each zone of intensity and specially cause an underestimation of the time spent above $\mathrm{VT}_{2}$.

In conclusion, the present results shows that power output associated with $\mathrm{VT}_{2}$ and MPT is reduced after a short training session. As a consequence, training intensities based on power output (associated with $\mathrm{VT}_{2}$ or in \%MPT) would be overestimated (i.e. higher metabolic state) after a short period of the training session. This casts doubt therefore, on the pertinence of using the power output (or running speed by extension) for prescription of exercise intensities during prolonged training sessions. In contrast, because $\mathrm{VO}_{2}$ associated with $\mathrm{VT}_{2}$ and MPT is unchanged after the same short training session, we could assume that exercise intensity based on $\dot{\mathrm{V}}_{2}$ would be more stable. Consequently, because the same exercise prescribed at $\mathrm{VT}_{2}$ using power output or $\dot{\mathrm{V}}_{2}$ would not induce the same metabolic intensity of training (and very certainly, different training adaptations), it questions which method (power output/running speed or $\dot{\mathrm{VO}}_{2}$ ) should be used to monitor training intensity. In other words, which is the best mean (power output/running speed or $\dot{\mathrm{V}}_{2}$ ) to monitor training intensity? Because heart rate (HR) measurements are often used by sportmen, it could also be interesting to study the effects of the same short training session on the HR associated to VTs and MPT. Overall, more research is needed to elucidate these crucial points for coaches and clinicians.

\section{Practical implications}

- Our results could explain that an exercise prescribed in respect to power output associated with $\mathrm{VT}_{2}$ or defined in \% of MPT would be difficult to perform at the end of a training session.

- Power output (or running speed by extension) should be used with caution for the study of distribution of training/competition intensities (in respect to $\mathrm{VT}_{2}$ or in \% of MPT) because it could certainly induce an underestimation of the physiological demand.

- Establish training/rehabilitation intensities with $\dot{\mathrm{V}}_{2}$ or power output (associated with $\mathrm{VT}_{2}$ or in \% of MPT) might not match the same exercise intensities (in term of physiological constraint) and thus would induce different training adaptations.

\section{Acknowledgments}

The authors are grateful to the subjects for having accepted to participate in this study. They also thank Dr. Thibault Deschamps and Fabien Cloud for their inputs.

\section{Appendix A. Supplementary data}

Supplementary data associated with this article can be found, in the online version, at doi:10.1016/j.jsams.2007.11.001.

\section{References}

1. Wasserman K, Mcllroy MB. Detecting the threshold of anaerobic metabolism in cardiac patients during exercise. Am J Cardiol 1964; 14:844-52.

2. Astrand PO, Rodahl K. Textbook of work physiology. New York: McGraw-Hill; 1985.

3. Davis JA, Frank MH, Whipp BJ, Wasserman K. Anaerobic threshold alterations caused by endurance training in middle-aged men. J Appl Physiol 1979;46(6):1039-46.

4. Lonsdorfer-Wolf E, Bougault V, Doutreleau S, Charloux A, Lonsdorfer J, Oswald-Mammosser M. Intermittent exercise test in chronic obstructive pulmonary disease patients: how do the pulmonary hemodynamics adapt? Med Sci Sports Exerc 2004;36(12):2032-9.

5. Lind E, Joens-Matre RR, Ekkekakis P. What intensity of physical activity do previously sedentary middle-aged women select? Evidence of a coherent pattern from phys- 
iological, perceptual, and affective markers. Prev Med 2005;40(4):407-19.

6. Pialoux V, Mounier R, Ponsot E, et al. Effects of exercise and training in hypoxia on antioxidant/pro-oxidant balance. Eur $J$ Clin Nutr 2006;60(12):1345-54.

7. Reinhard U, Muller PH, Schmulling RM. Determination of anaerobic threshold by the ventilation equivalent in normal individuals. Respiration 1979;38(1):36-42.

8. Buchfuhrer MJ, Hansen JE, Robinson TE, Sue DY, Wasserman K, Whipp BJ. Optimizing the exercise protocol for cardiopulmonary assessment. J Appl Physiol 1983;55(5):155864.

9. Myers J, Ashley E. Dangerous curves. A perspective on exercise, lactate, and the anaerobic threshold. Chest 1997;111(3):787-95.

10. Caiozzo VJ, Davis JA, Ellis JF, et al. A comparison of gas exchange indices used to detect the anaerobic threshold. $J$ Appl Physiol 1982;53(5):1184-9.

11. Seiler KS, Kjerland GO. Quantifying training intensity distribution in elite endurance athletes: is there evidence for an "optimal" distribution? Scand J Med Sci Sports 2006; 16(1):49-56.

12. Chicharro JL, Hoyos J, Lucia A. Effects of endurance training on the isocapnic buffering and hypocapnic hyperventilation phases in professional cyclists. $\mathrm{Br} J$ Sports Med 2000;34(6):450-5.

13. Duffield R, Dawson B, Pinnington HC, Wong P. Accuracy and reliability of a Cosmed K4b2 portable gas analysis system. J Sci Med Sport 2004;7(1):11-22.

14. McLaughlin JE, King GA, Howley ET, Bassett Jr DR, Ainsworth BE. Validation of the COSMED K4 b2 portable metabolic system. Int J Sports Med 2001;22(4):280-4.

15. Carter $H$, Jones AM, Barstow TJ, Burnley M, Williams CA, Doust JH. Oxygen uptake kinetics in treadmill running and cycle ergometry: a comparison. J Appl Physiol 2000;89(3):899-907.

16. Black A, Ribeiro JP, Bochese MA. Effects of previous exercise on the ventilatory determination of the aerobic threshold. Eur J Appl Physiol Occup Physiol 1984;52(3):315-9.

17. Raymer GH, Forbes SC, Kowalchuk JM, Thompson RT, Marsh GD. Prior exercise delays the onset of acidosis during incremental exercise. J Appl Physiol 2007;102:1799805.
18. Marles A, Mucci P, Legrand R, Betbeder D, Prieur F. Effect of prior exercise on the $\mathrm{VO}_{2}$ / work rate relationship during incremental exercise and constant work rate exercise. Int J Sports Med 2006;27(5):345-50.

19. Hagberg JM, Coyle EF, Carroll JE, Miller JM, Martin WH, Brooke MH. Exercise hyperventilation in patients with MCArdle's disease. J Appl Physiol 1982;52(4):991-4.

20. Hug F, Faucher M, Marqueste T, Guillot C, Kipson N, Jammes $\mathrm{Y}$. Electromyographic signs of neuromuscular fatigue are concomitant with further increase in ventilation during static handgrip. Clin Physiol Funct Imaging 2004;24(1):25-32.

21. McCloskey DI, Mitchell JH. Reflex cardiovascular and respiratory responses originating in exercising muscle. $J$ Physiol 1972;224(1):173-86.

22. Smith SA, Gallagher KM, Norton KH, Querry RG, WelchO'Connor RM, Raven PB. Ventilatory responses to dynamic exercise elicited by intramuscular sensors. Med Sci Sports Exerc 1999;31(2):277-86.

23. Meyer T, Kindermann M, Kindermann W. Exercise programmes for patients with chronic heart failure. Sports Med 2004;34(14):939-54.

24. Gimenez M, Servera E, Vergara P, Bach JR, Polu JM. Endurance training in patients with chronic obstructive pulmonary disease: a comparison of high versus moderate intensity. Arch Phys Med Rehabil 2000;81(1):102-9.

25. Londeree BR. Effect of training on lactate/ventilatory thresholds: a meta-analysis. Med Sci Sports Exerc 1997;29(6):837-43.

26. Dufour SP, Ponsot E, Zoll J, et al. Exercise training in normobaric hypoxia in endurance runners. I. Improvement in aerobic performance capacity. J Appl Physiol 2006; 100(4):1238-48.

27. Lepretre PM, Lopes P, Koralsztein JP, Billat V. Fatigue responses in exercise under control of $\mathrm{VO}_{2}$. Int J Sports Med, in press.

28. Laursen PB, Jenkins DG. The scientific basis for highintensity interval training: optimising training programmes and maximising performance in highly trained endurance athletes. Sports Med 2002;32(1):53-73.

29. Ebert TR, Martin DT, McDonald W, Victor J, Plummer J, Withers RT. Power output during women's World Cup road cycle racing. Eur J Appl Physiol 2005;95(5-6):529-36. 